\title{
MedienPädagogik
}

Zeitschrift für Theorie und Praxis der Medienbildung

Jahrbuch Medienpädagogik 3.

Zweitveröffentlichung aus: Jahrbuch Medienpädagogik 3. (2003) Opladen: Leske + Budrich. Herausgegeben von Ben Bachmair, Peter Diepold und Claudia de Witt.

\section{Neue Medien im Kontext der pragmatistischen Bildungstheorie}

\author{
Claudia de Witt
}

Die Bedeutung des mediengestützten Lernens nimmt in allen Bildungskontexten zu, wie in Schule, Hochschule und Weiterbildung. Neuen Medien wird eine entscheidende Rolle bei der Veränderung des Lehrens und Lernens zugesprochen. Die Faszination dieser Medien liegt z.B. darin, dass sie mit ihrem Distributionspotenzial über Lernressourcen mit Datenbanken, Lernmaterialien, Expertenwissen verfügen. Sie zeichnen sich aber auch durch ihr Interaktions- und Kommunikationspotenzial aus, mit dem sie die Organisation zeit- und ortsflexibler Lernprozesse beeinflussen und verändern (vgl. Hesse/Friedrich 2001). In Lehr- und Lernprozessen können neue Medien drei Funktionen übernehmen: die Funktion als Wissenswerkzeug sowie ihr Einsatz zur Wissensrepräsentation und zur Wissensvermittlung. ${ }^{1}$ Angesprochen sind damit Multimedia-Dokumente, HypertextStrukturen und Netzwerkdienste. Andererseits setzen neue Medien und insbesondere das Internet die gesellschaftlichen, sozialen und kulturellen Differenzierungs- und Individualisierungstendenzen fort. Neue Medien wie das Internet verändern Menschen in ihrem Denken und Handeln, beeinflussen gesellschaftliche Institutionen. Diese Veränderungsprozesse betreffen die Perspektive von Bildung. Betrachtet man die aktuelle medienpädagogische Forschungslandschaft, so lässt sich eine Polarisierung ausmachen, auf die Marotzki (2000) hingewiesen hat: nämlich die Polarisierung der medienpädagogischen Thematik mit lerntheoretischen Implikationen einerseits und bildungstheoretischen Überlegungen andererseits.

Eine Auseinandersetzung mit neuen Medien zur Unterstützung von Lernprozessen muss Bildungsprobleme in den Blick nehmen. Medien sollen nicht nur das Lehren und Lernen verbessern, sondern bei der Lösung bestimmter Bildungsprobleme helfen (vgl. Kerres 2001). Hinzu kommt, dass es eine

\footnotetext{
1 Als Wissenswerkzeug unterstützen die neuen Medien die Konstruktion und Kommunikation von Wissen; bei der Wissensrepräsentation geht es um die Darstellung und Organisation von Wissen; in Bezug auf die Wissensvermittlung tragen die neuen Medien zur Steuerung und Regelung des Lernprozesses bei (vgl. Kerres 2001, S. 94)
} 
kaum zu überblickende Fülle an Einzelergebnissen zur empirischen Medienforschung gibt. Aber äußerst selten sind pädagogische Grundpositionen zu finden, von denen Bildungs-, Erziehungs- und Lernprozesse unterstützt werden können. Es bedarf theoretischer Bezugsrahmen, um medienpädagogische Aufgaben im Verhältnis zu anderen Erziehungs- und Bildungsaufgaben richtig zu gewichten (vgl. Spanhel 1997).

Impulse für Innovationen medienpädagogischer Konzeptionen können aus zwei Richtungen kommen: entweder aus der medialen Praxis oder aus der medienpädagogischen Theorie. Für Innovationen im Bereich des Lehrens und Lernens mit neuen Medien sind medienpädagogische Ansätze geeignet, die bereits die Verbindung von Theorie und Praxis berücksichtigen und bildungsphilosophische Grundlagen aufnehmen. Aus dem bildungstheoretischen Blickwinkel ergeben sich dann Impulse, die Praxis des Lehrens und Lernens mit neuen Medien weiterzuentwickeln. Damit werden Aspekte einer Medienpädagogik erarbeitet, die die Reflexion über und den Umgang mit neuen Medien fortsetzt und zu einer innovativen pädagogischen und gestaltungsorientierten Praxis mit neuen Medien führt.

In diesem Beitrag wird eine bildungstheoretische Sicht der Medienpädagogik gewählt, von der aus die Möglichkeiten der neuen Medien für Bildungs- und Lernprozesse neu betrachtet werden können. Der theoretische Bezugsrahmen ist der amerikanische Pragmatismus nach John Dewey.

Man kann sich natürlich sofort die Frage stellen, wie ausgerechnet pragmatistisches Denken hilft, Probleme der Medienbildung zu bewältigen. Medien und neue Technologien gelten als besonders dynamische Wissensgebiete und mit dem Wissen wächst der Bedarf zur Bewältigung dieser Informationsund Wissensmengen. Gerade im Hinblick auf die Bildungsanliegen des selbstgesteuerten und lebenslangen Lernens mit neuen Medien sind Ansätze gefragt, die eine kontinuierliche Bewältigung und Aneignung von Wissen berücksichtigen.

Auch um die pädagogische Bedeutung der Medien klarer zu erkennen und besser zu verstehen, wird die Denkstruktur der pragmatistischen Bildungstheorie neu skizziert. Diese Denkfigur kann zu einem neuen Verständnis medienpädagogischer Theoriebildung und medialer Praxis beitragen. Der Denkansatz läuft auf die Begründung eines medienpädagogischen Ansatzes hinaus, in dem Medienbildung als ein inhaltlich orientierter Methodenprozess zu begreifen ist (vgl. de Witt 1999). Die Methode ist dabei nicht nur ein Mittel im pädagogischen Prozess, sondern der pädagogische Prozess selbst und wird über die Rekonstruktion von Erfahrung beschrieben. Bevor aber der pragmatistische Bildungsbegriff erläutert wird, werden die Anforderungen des selbstgesteuerten Lernens mit neuen Medien dargestellt. 


\section{Neue Medien und selbstgesteuertes Lernen}

Netzbasierte Lernumgebungen können selbstgesteuertes und selbstverantwortliches Lernen unterstützen. Während sich in fremdgesteuerten Lernumgebungen „die meisten Aktivitäten zur Anregung von Lernprozessen auf einen Lehrenden bzw. seine mediale Repräsentation konzentrieren“ (Wilbers 2001, S. 15), übernimmt in selbstgesteuerten Lernumgebungen der Lernende selbst diese Funktion. Für Knowles ist selbstgesteuertes Lernen ein Prozess, bei dem „der Lerner - mit oder ohne Hilfe anderer - initiativ wird, um seine Lernbedürfnisse festzustellen, seine Lernziele zu formulieren, menschliche und dingliche Ressourcen für das Lernen zu identifizieren, angemessene Lernstrategien zu wählen und zu realisieren und um Lernergebnisse zu evaluieren“ (Knowles 1980, zit. und übers, von Friedrich 2000, S. 3).

Außerdem weist Friedrich mit Bezug auf andere Autoren wie Simons hin, dass eine Selbststeuerung ohne jegliche Fremdsteuerung in der Praxis selten vorkommt. „Selbst Autodidakten setzen sich mit dem Griff zum Lehrbuch oder zur Ratgeberliteratur ... einer mehr oder minderen Form der Fremdsteuerung aus“ (Friedrich 2000, S. 4). Vielmehr, so Simons, handle es sich um ein Kontinuum, das sich zwischen zwei Extremen erstreckt: zwischen dem völligen Unvermögen, das eigene Lernen zu steuern und zu kontrollieren, und der Fähigkeit, dies ganz ohne externe Hilfe zu tun. In ihrer Reinform kommen aber beide Extreme fast nicht vor" (Simons 1992, S. 251; zit in Friedrich 2000, S. 4). In der Praxis des Lernens sei ein Mischungsverhältnis zwischen Selbst- und Fremdsteuerung erfolgreich.

Selbstgesteuertes Lernen erfordert ein hohes Maß an Selbstorganisation und Selbstreflexion und ist ein komplexes Phänomen. Beim selbstgesteuerten Lernen „müssen Entscheidungen über Lernziele (woraufhin), über Inhalte (was), über Lernressourcen (Medien, Lernmittel), über zeitliche Aspekte (wann), über methodische Aspekte (mentale Verarbeitung des Lerninhalts), über die Art und Weise der Feststellung der Lernzielerreichung (Evaluation) getroffen werden“ (Friedrich 2000, S. 3). Um diese Komplexität des Prozesses konkreter zu verdeutlichen, stellt Wilbers mit Bezug auf Simons die Lernfähigkeiten zusammen, die mit einer selbstgesteuerten Lernumgebung gefördert bzw. gefordert werden:

- „Lernen vorbereiten können“

- „Lernhandlungen ausführen können“,

- „Lernhandlungen regulieren können“

- „Leistungen bewerten können“ (Wilbers 2001, S. 16).

Es gibt eine Reihe simulativer Methoden, die das selbstgesteuerte Lernen unterstützen und Praxisnähe herstellen: Dazu zählen Planspiele, die Simulationen von Entscheidungsprozessen verkörpern, Fallstudien, in denen authentische Situationen entlang einer Geschichte mit problematischen Ereignissen beschrieben werden, oder Webquests. Bei Webquest z.B. handelt es sich um eine besondere Art der Fallmethode, die nur über das Internet bear- 
beitet wird. Ein Webquest gehört zu den Online-Assignments, lernerzentrierten Methoden, die den Teilnehmern die Möglichkeit zum selbstgesteuerten Lernen geben, indem sie komplexere Aufgabenstellungen lösen müssen und Lernmaterialien heranziehen. Besonders die Informationsbeschaffung spielt eine große Rolle, Informationsquellen werden dafür aus dem Internet angeboten, Experten sind über Mail oder Conferencing ansprechbar, Vorgehensweisen werden empfohlen, die bei der Lösung des Problems sinnvoll erscheinen. Zur Bewertung der Aktivitäten stehen den Lernenden Bewertungskriterien, Gruppen- und Einzelbewertungen zur Verfügung. Individuelles Feedback und problemspezifische Hilfestellungen liefert ein Online-Tutor. Da die Lernenden aktiv sind, sich mit einer Thematik intensiv auseinander setzen, selbständig Ressourcen suchen und analysieren müssen, können sie sich so ein gewisses Maß an Orientierungswissen aneignen (vgl. Seufert/Back 2001).

Ein Webquest ist demnach eine forschungsorientierte Aktivität, bei der der Lernende alle Informationen aus Quellen des Internets bezieht. Ein Webquest besteht aus folgenden Elementen: Einer Einführung, Aufgaben mit unterschiedlichen Anforderungsniveaus, einer Reihe von Informationsquellen, einer Beschreibung der Herangehensweise und einer Zusammenfassung. Nach diesem Webquest ist ein Wissensbereich tiefgehend analysiert und transformiert. Das erworbene Wissen wird über das Materials demonstriert, indem der Lernende etwas produziert, auf das die anderen entweder offoder online reagieren und antworten. Bernie Dodge und Tom March hatten die Idee zu Webquests 1995 an der San Diego State University (http://edweb.sdsu.edu/webquest/ webquest.html).

Simulative Methoden wie Planspiele oder Webquest versetzen den Lernenden in die Situationen eines Entscheiders und zielen auf den Erwerb von Kompetenzen mit komplexen Systemen. Damit setzen sie in netzbasierten Lehr- und Lernsituationen das um, was auf der Grundlage einer Bildungstheorie gefordert wird, nämlich dass der Lernende Wissen kontinuierlich anwendet und dieses sich dadurch in der Praxis auch bewährt, und dass der Lernende sein Wissen nur durch diese Praxis rekonstruktiv weiter verarbeitet. Damit komme ich zu den bildungstheoretischen Ansprüchen an ein Lernen mit neuen Medien.

\section{Die Perspektive der pragmatistischen Bildungstheorie}

Der amerikanische Pragmatismus entwickelte sich in der 2. Hälfte des 19. Jahrhunderts durch Charles Peirce, William James, George H. Mead und John Dewey. Das Aufkommen des Pragmatismus war eine Konsequenz aus den Problemen, die durch die neuen Strukturen der Industriegesellschaft entstanden und zur Notwendigkeit praktischer Problemlösungsprozesse führten. So wurde kritisiert, die Industrialisierung habe dazu geführt, dass erstens bei vielen Menschen ein deutlicher Mangel an Primärerfahrung sichtbar gewor- 
den sei und dass zweitens die zwischenmenschlichen Beziehungen nachhaltig und empfindlich gestört worden seien. Nicht umsonst hebt John Dewey $(1859$ - 1952) den Demokratiegedanken und die Kommunikationssituation in seinen Werken hervor.

Als einer der Gründungsväter des Pragmatismus hat er das pragmatistische Denken in pädagogische Zusammenhänge gebracht. Der Pragmatismus (vom griech. „pragma“: das Getane; aber auch das, was zu tun ist; das Handeln; das Tun, die Tätigkeit) ist eine Wissenschaftstheorie, die hinter der Pädagogik Deweys steht und theoretisch angeleitete Handlungen in das Zentrum der Betrachtung rückt. Der Pragmatismus vertritt also eine handlungstheoretische Auffassung von Wissenschaft. Dementsprechend sind mit „pragmatistisch“ solche Handlungsprozesse gemeint, die von vernünftigen Denkprozessen gesteuert werden. Denken, Fühlen, Wollen, Werten haben ihre Funktion im lebensbestimmenden Handeln. Dewey geht in seiner Philosophie und Pädagogik von dem Gedanken aus - der für uns heute fast selbstverständlich klingt -, dass Theorie und Praxis in einem engem Zusammenhang stehen und „zeigt, wie die Theorie sich aus der Praxis heraus entwickelt, wie die Theorie in Form von Hypothesen zeitweise eine gewisse Selbständigkeit gegenüber der Praxis behauptet, um dann schließlich Teil der Praxis zu werden, sich dort zu bewähren und in der Praxis eine Leitfunktion zu übernehmen. ... Wenn immer möglich, beginnt der einzuschlagende Weg bei der Praxis und führt über die Theorie zur Praxis zurück“ (Schäfer 1988, S. 5f.).

\subsection{Einige Missverständnisse über den Pragmatismus}

Mit den pragmatistischen Grundstrukturen kann eine Standortbestimmung von Erkenntnis und Wissen vorgenommen werden, die auch in der heutigen Informations- und Wissensgesellschaft angemessene Richtlinien enthält. Aber mit seiner Anerkennung hat es der Pragmatismus nicht immer leicht gehabt. Der amerikanische Pragmatismus insbesondere von John Dewey wurde und wird sehr häufig für pädagogische Schlagworte verwendet, dabei aber auch oft missverstanden und zu Unrecht abgelehnt. Ich möchte hier nur drei große Missverständnisse nennen:

Ein Missverständnis kommt daher, dass

1. Deweys Pädagogik nicht als Ganzes betrachtet wurde, sondern nur partiell und von daher oft einseitig. Ein großes Missverständnis entstand eben dadurch, dass in vielen deutschen Rezeptionsversuchen nicht auf Deweys eigene philosophische Entwicklung Rücksicht genommen wurde und das Gesamtfeld des Pragmatismus nicht erschien. Z.B. wurde der Pragmatismus als unbedeutende Zusatzmethode in den unteren Volksschulklassen angesehen, in der Kaiserzeit z.B. bei Wilhelm Münch (vgl. Bittner 2001). Demokratische Zielvorstellungen wie „self-direction“ und 
2. „self-realization“ wurden von Dewey schon formuliert, aber sie wurden nicht erkannt oder weitergegeben (vgl. ebd.). ${ }^{2}$

3. Eine Interpretation wird Dewey auch nicht gerecht, die ihm - wie z.B. in der (reform-)pädagogischen Rezeption - nur den Vorrang einer Schülerzentrierung einräumt Dewey hat sich auch in seinen frühen Schriften niemals dafür ausgesprochen, „dass der Lernende wichtiger wäre als der Gegenstand oder der Lehrer. Ihm ging es vielmehr um ein Ausbalancieren der drei Seiten“ (Lehmann-Rommel 2001, S. 138).

4. Das dritte Missverständnis: Die pragmatistische Pädagogik wurde immer mit „learning by doing "verbunden. Irrtümlich ist diese Aussage zum Markenzeichen seiner Pädagogik avanciert. Denn 1. ist es gar nicht nachgewiesen, dass die Aussage von Dewey kommt und es ist 2. eine viel zu große Verkürzung dessen, was hinter der pragmatistischen Bildungstheorie steht (vgl. Bittner 2001).

Außerdem ist es aus der Sicht des Pragmatismus eine Verkürzung, dass Erfahrung häufig nur ein planloses Element in der Entwicklung des Menschen ist. Der Begriff der Erfahrung ist viel komplexer und enthält im Grunde die pragmatistische Vorstellung von Bildung. Deshalb bedeutet Erfahrung hier nicht nur das Ergebnis von Handlungen, sondern diese Vorstellung beinhaltet ebenfalls und gleichwertig den Prozesscharakter von Erfahrung. Damit wird der methodische Charakter von Bildung bereits angedeutet, der nun aus den weiteren Überlegungen genauer erschlossen wird.

\subsection{Die pragmatistische Bildungstheorie neu denken}

Bisher wurde wenig beachtet, dass die pragmatistische Bildungstheorie auch „postmodeme“ Elemente verwendet. Dazu gehört zum einen die Akzeptanz partikularer Positionen, zum anderen eben auch die Kategorien der Kontingenz und der Relationen. Die pragmatistische Bildungstheorie, so wie sie hier verstanden wird, begreift Bildung als einen methodischen Prozess. Der Methodenbegriff setzt auf den Prozess, die transversale Relationalität und die Kontinuität von Erfahrung und ist daher bestens geeignet, als Basis einer Medienbildung zu fungieren. Denn Bildungsziele in der Informationsgesellschaft können nicht mehr nur von Dauerhaftigkeit ausgehen, sondern müssen ständige Erneuerungsprozesse einschließen (vgl. de Witt 1999).

Dies kann daraus hergeleitet werden, dass es dieser Bildungstheorie nicht um das Streben nach Idealen, nicht um das Festlegen von absoluten Zielen, sondern um einen ständigen Veränderungsprozess geht, der durch Interaktio-

2 Genauso richtete sich das Interesse an Dewey ab 1965 hauptsächlich auf schul- und unterrichtspraktische Darstellungen. „Dort wird er zu Problemstellungen einer Schultheorie, des politischen Unterrichts, des problemlösenden Lernens, des Projektunterrichts oder zu fachdidaktischen Fragestellungen herangezogen“ (Bittner 2001, S. 221) und mit der Projektmethode in Verbindung gebracht. 
nen und Kontinuitäten gestaltet wird und durch den sich bildende Erfahrung rekonstruiert. Als Methode verstanden unterliegt die Bildungstheorie nicht einer Regelhaftigkeit, sondern impliziert einen individuell auslegbaren Denkweg, der sich im Wesentlichen am Ansatzpunkt der Erfahrung festmacht.

Dieser Denkweg beginnt mit der Ablehnung von Dualismen. Und damit gibt es in der pragmatistischen Denkweise keine Hierarchisierung von Zielen vor Mitteln, von Produkten vor Prozessen. Eine Ergebnis- bzw. Zielorientierung z.B. wird nicht der Prozess- bzw. Mittelorientierung vorgezogen. Hierarchisierungen werden vermieden, weil sie generell immer mit einer Instrumentalisierung verbunden sind und damit gleichzeitig auch Abwertungen mit einschließen. Die pragmatistische Medienbildungstheorie baut deshalb auf folgenden Voraussetzungen auf:

- Situationen als Ausgangspunkt für Erfahrungen,

- Prozesshaftigkeit und Kontinuität medialer Erfahrung,

- Denken in Relationen.

\section{Situationen als Ausgangspunkt für Erfahrungen}

Eine pragmatistische Medienpädagogik orientiert sich an den menschlichen Situationen, die durch ihre singulären Ereignisse Kontingenzen, Zufälligkeiten, unvorhersehbare Möglichkeiten einschließen. Und nur dadurch, dass es Kontingenzen gibt, die in einzigartigen Situationen und Tätigkeiten verankert sind, ist die Freiheit des Handelns möglich. Denn der Mensch hat die Möglichkeit Reflexionsprozesse in Gang zusetzen, um der Instabilität und Kontingenz von Welt zu begegnen und sich in den Cyberwelten zurechtzufinden.

\section{Prozesshaftigkeit und Kontinuität medialer Erfahrung}

Was das Paradigma des Netzes als Differenz und Konnektion bezeichnet, wird hier pädagogisch mit den Begriffen der Prozesshaftigkeit bzw. Interaktivität und Kontinuität von Erfahrung weitergeführt. Denn die Situationen im Netz verlangen die Fähigkeit, sich in nicht-sequentiellen Strukturen zurechtzufinden und trotzdem Dinge in Beziehung zu setzen. Vereinheitlichung und Differenzierung werden gleichermaßen erfahren. Erfolgreiches und sinnvolles Handeln mit neuen Medien ist daher wesentlich vom Umgang mit hypermedialen Strukturen, mit „Knoten“ („,nodes“) und „Verknüpfungen“ („links“) abhängig. Im Internet erschließt sich - im Gegensatz zum linearen Medium Film - die Bedeutung erst durch die Interaktion von Nutzer und Medium, durch die eingreifende Handlung des Nutzers.

Ein ständiger Veränderungsprozess bedeutet aber nicht einen Bruch mit der Kontinuität der Erfahrung, sondern im Gegenteil: Gerade nur durch die Verbindungen der neu erfahrenen Elemente mit den alten entsteht eine Kontinuität. Kontinuität bedeutet, dass Erfahrung ebenso von den vorangegange- 
nen Erfahrungen beeinflusst wird, wie sie ihrerseits die Qualität der nach ihr folgenden Erfahrungen modifiziert. Die vorangegangenen Erfahrungen verändern sich durch die Verbindung mit den neuen Erfahrungen und validieren sich möglicherweise. So kann man etwas Neues nicht begreifen ohne die Ideen und Kenntnisse, die man bereits besitzt. Das Alte, Bekannte verändert sich ebenfalls, „wenn es dazu verwendet wird, das Neue zu erfassen und zu interpretieren“ (Dewey 1995, S. 81). Die Konnektionen können zu immer neuen und anderen Resultaten aber nur durch bewusst vollzogene Handlungen gelenkt werden. Man muss Hypothesen und Theorien haben, um seine Kenntnisse zu einer bildenden Erfahrung zu organisieren.

\section{Denken in Relationen}

Der Umgang mit dem Internet verstärkt die Gewissheit, dass ein Denken in universalen Ansprüchen obsolet ist, ein Denken in Dualismen zur Einseitigkeit neigt, ein Denken in Relationen aber der Offenheit von Welt eher entspricht. Das Denken in Relationen lässt zu, dass Unterschiede gewahrt bleiben und gleichzeitig Verbindungen und Übergänge bedacht werden. Dewey lehnt konsequenterweise die idealistisch geprägte Hierarchisierung eines denkenden Zugangs zur Welt vor der gewöhnlichen Erfahrung nämlich ab, weil es falsch sei, „dass der erste Zugang zur Realität der des Denkens ist“ (LehmannRommel 2001, S. 140f.).

Warum ist Deweys Kritik gerechtfertigt? Die Fixierung auf Dualismen wie z.B. Subjekt versus Objekt, Individuum versus Gesellschaft, Geist versus Körper ist dadurch zustande gekommen, weil der situative Kontext und das subjektive Eingebundensein in der jeweiligen Situation vernachlässigt wurde. Wenn man von den Situationen ausgeht, in denen Interaktionen stattfinden, können Spannungen, Widersprüche, Kontingenzen nicht nur im Denken aufgelöst werden mit dem Ziel durch Reflexion alleine eine Einheit herzustellen. Situationen schließen durch ihre singulären Ereignisse Kontingenzen, Zufälligkeiten, unvorhersehbare Möglichkeiten immer mit ein. Ein Handeln in der situativen Praxis kann also keine absolute Gewissheit gewährleisten. Deweys Alternative zur dualistischen Denkform ist also prozess- und interaktionsorientiert, indem er auf den interaktiven Prozess des Handelns verweist, durch den Spannungen, Widersprüche und Kontingenzen entgegnet und bearbeitet werden. Dieser situativen Praxis angemessener ist demnach ein Denken in Relationen. Das heißt z.B., dass absichtsvolles Handeln nicht dem Subjekt allein zugeordnet ist, sondern sein Handeln in Relation zum jeweiligen Kontext gesehen werden muss. Das Denken in Relationen ist also die Konsequenz daraus, dass der situative Handlungsbezug mit seinen Spannungen und Kontingenzen die Grundlage des Erfahrungsprozesses ist (vgl. de Witt 1999).

Um das Denken in Relationen noch einmal zu verwenden: Der situative Handlungsbezug hat Konsequenzen für die Relation des vergangenen, gegenwärtigen und zukünftigen Handelns: Gegenwärtiges Handeln kann nur 
dann beurteilt und sein Sinn abgeschätzt werden, wenn die Vergangenheit und die Zukunft mit gedacht werden. Das heißt aber nicht, dass die Zukunft gegenüber dem gegenwärtigen Handeln den Vorrang erhält. „Zukunft ist nur Hilfsquelle für sinnvoll erfahrene Gegenwart“, so Dewey (1931, S. 276). Dies bedeutet nämlich eine gleichwertige Anerkennung der Zufälligkeiten und relationalen Zusammenhänge, unter denen das gegenwärtige Handeln stattfindet. Die Freiheit des Handelnden liegt darin, „die Möglichkeiten der Gegenwart für das eigene Zielverfolgen auszuschöpfen“ (Lehmann-Rommel 2001, S. 150). Ein Einlassen auf Kontingenzen in den verschiedenen Kontexten der Erfahrung schafft damit Raum für ein experimentierendes Denken und Handeln im Rahmen eines lebenslangen Lernprozesses.

Damit nähern wir uns der wesentlichsten Erkenntnis bei der Neuformulierung des pragmatistischen Bildungsbegriffs: Es betrifft den Stellenwert des Methodischen in dieser Theorie. Denn bisher wurde verkannt, dass Bildung nicht nur das Ergebnis eines Lernprozesses ist sondern der Lernprozess selbst. Dabei wird nicht die Methode gegen den Inhalt ausgespielt.

Das Innovative und damit der besondere Blick des Methodischen in der pragmatistischen Bildungstheorie lassen sich über den Begriff des Rekonstruierens erschließen. Der Bildungsprozess ist „eine ständige und kontinuierliche Rekonstruktion von Erfahrung, ein dauernder Neuaufbau, eine ständige Reorganisation“ (Dewey 2000, S. 75), Prozess und Ziel der Bildung sind ein und dieselbe Sache, Prozess der Erfahrung ist identisch mit dem Ergebnis von Erfahrung. Erfahrung wird damit nicht als planloser Vorgang beschrieben.

An die Stelle von außerhalb der Handlung gesetzten Zielen setzt Dewey den relationalen Charakter von Zielen, Mitteln und Konsequenzen im Prozess der Erfahrung. Da dieser die Möglichkeit von Kontingenzen einschließt, werden in diesem aktiven Vorgang situationsbezogen rückwärts und vorwärts Bedeutungen zwischen Zielen, Mitteln und Ergebnissen unseres Handelns hergestellt. „Es bedeutet, dass die Erfahrung als ein aktiver Vorgang ... Zeit erfordert, und dass die späteren Teile dieses Vorgangs die früheren vervollständigen; die späteren Teile bringen Beziehungen ans Licht, die in den früheren bereits vorhanden und wirksam, aber noch nicht erkannt waren“ (Dewey 2000, S. 111). Zentraler Bestandteil des methodischen Bildungsverständnisses ist deshalb die Rekonstruktion von Erfahrung. Der Erfahrungsprozess ist gleichsam die Methode mit Realität umzugehen, dadurch erkenntnisproduzierend und nicht dogmatisch.

\section{Der Stellenwert neuer Medien in der pragmatistischen Bildungstheorie}

Ein theoretischer Bezugsrahmen weist den Medien einen systematischen Stellenwert in einem Begriff von Bildung zu. Die Konsequenz aus der pragmatistischen Bildungstheorie für die Medienpädagogik ist, dass es auf die 
Situationen ankommt, die den Wert eines Mediums bestimmen, und nicht auf das Medium selbst. So wie neue Medien nur dann sinnvoll genutzt werden können, wenn sich Lernprozesse verändern, so kommt es auch darauf an, ein Medium in Relation zu einem Bildungsprozess und Bildungsproblem zu betrachten (vgl. Kerres 2001). Mit der Forderung nach selbstgesteuertem und lebenslangem Lernen wird ein Bildungsbegriff anvisiert, der vom Einzelnen eine ständige Rekonstruktion medialer Kontexte verlangt. Der Lernende (re-) konstruiert durch seine gewählten Lernpfade im Internet Muster von Konnektionen. Lernen findet dabei nicht allein in den Begriffen der Handlung oder nur in Begriffen des Inhalts statt. Lernen wird über die Handlung als authentische Aktivität definiert, die in Relation zu dem Inhalt steht. Der Lernprozess beinhaltet dann z.B. die Fähigkeit kritisch in den Inhaltsbereichen zu denken, Informationen zu beschaffen, die in Relation zu einem Problem stehen und sie dann über eine Reflexionsphase zur Geltung zu bringen.

Warum wird hier der Ansatz einer pragmatistischen Medienbildung verfolgt, der auf der Theorie von John Dewey basiert? Der pragmatistische Bildungsansatz ist überzeugend, weil er an der Lernsituation als Ausgangspunkt für die Gestaltung multimedialer, internetbasierter Lernumgebungen ansetzt. Deshalb kommen Planspielen, Fallstudien und Webquest mit ihrer simulativen Praxis der Umsetzung dieser Bildungsidee am nächsten. Medien sind nicht nur als Instrumente anzusehen, sondern ihre Bedeutung erhalten sie in der jeweiligen Lernsituation. Bei der Gestaltung internetbasierter selbstgesteuerter Lernumgebungen ist die gesamte Lernorganisation zu berücksichtigen.

Beim E-Learning sind Lernende z.B. in Aktivitäten zu verstricken, indem sie die Konsequenzen ihres Handelns antizipieren lernen und verantwortungsvolle Entscheidungen treffen - sei es durch entsprechende Lernaufgaben (Fälle), Kommunikationsformen, das Eingebundensein in Konkurrenzsituationen. Es bedarf noch einer größeren Vielzahl an solchen simulativen Lernumgebungen, die das selbstgesteuerte und lebenslange Lernen fördern.

Aufgabe medienpädagogischen Handelns ist die Gestaltung medialer Situationen, die zu solchen Prozessen des Erfahrens beitragen, die die Fähigkeiten fördern

- in offenen Prozessen der Interaktion Fähigkeiten zur Selbstständigkeit und Selbstorganisation und gleichzeitig kommunikative Kompetenzen zu entwickeln,

- Potentiale im Vorhandenen wahrzunehmen und

- die Konsequenzen des Handelns zu überblicken.

Pragmatistische Medienbildung kann dann dem Einzelnen eine Orientierung in einer Gesellschaft geben, die an ihn Ansprüche hinsichtlich Vielfältigkeit, Flexibilität, Spontaneität und enormer Leistungsfähigkeit stellt. Medienbildung setzt sich damit auch mit der Qualifizierung neuer Medien als Bildungsmedien auseinander, die den Nachweis erbringen müssen, dass mit diesen Medien Prozesse angeregt werden können, die deutlich machen, dass die Aneignung von Wissen gleichsam von Unsicherheiten und Risiken begleitet 
wird. Pragmatistisches Denken sollte Eingang finden in mediendidaktische Gestaltungsprozesse, um eine einseitige Theorievermittlung oder eine beliebige Praxisorientierung zu vermeiden. Der Einfluss der pragmatistischen Denkweise und Bildungstheorie auf didaktische Entscheidungen wirkt sich beispielsweise auf eine Auflösung von Hierarchisierungen aus. Die Konsequenz ist, dass Lernziele und Mittel jeweils von der aktuellen Lernsituation gedacht und dort in Beziehung gesetzt werden müssen.

Neben lerntheoretischen und kulturtheoretischen Aspekten spielen in der erziehungswissenschaftlichen Online-Medienforschung weiterhin bildungstheoretische Aspekte eine große Rolle. Ihre Zukunftsaufgaben liegen auch in einer stärkeren Verbindung von Bildungsproblemen und mediendidaktischer Prozessplanung und damit in einer stärkeren Verbindung von Medienbildung und Mediendidaktik.

\section{Literatur}

Bittner, St.: Leaming by Dewey? John Dewey und die deutsche Pädagogik 1900-2000. Bad Heilbrunn 2001.

Dewey, J.: Die menschliche Natur, ihr Wesen und ihr Verhalten. Stuttgart 1931. („Human Nature and Conduct“, MW 14:1922).

Dewey, J.: Erfahrung und Natur. Frankfurt 1995 („Experience and Nature“, LW 1:1925).

Dewey, J.: Demokratie und Erziehung. Weinheim 2000 („Democracy and Education“, MW 9:1916).

Friedrich, H.F.: Selbstgesteuertes Lernen - sechs Fragen, sechs Antworten. Soest 2000. (http://www. leam-line.nrw.de/angebote/selma/medio/vortraege/friedrich/friedrich.pdf, 22.3.2000).

Hesse, F.W./Friedrich, H.F.: Partizipation und Interaktion in virtuellen Seminaren - ein Vorwort. In: Hesse, F.W./Friedrich, H.F. (Hrsg.): Partizipation und Interaktion in virtuellen Seminaren. Münster 2001, S. 7-12.

Hesse, F.W./Friedrich, H.F. (Hrsg.): Partizipation und Interaktion in virtuellen Seminaren. Münster 2001,

Hohenstein, AVWilbers, K. (Hrsg.): Handbuch E-Learning. Köln 2001.

Kerres, M: Multimediale und telemediale Lernumgebungen. Oldenbourg 2001.

Lehmann-Rommel, R.: Deweys Aufhebung der dualistischen Denkform. Konsequenzen für den Umgang mit Zielen in der Pädagogik. In: Schreier, H. (Hrsg.): Rekonstruktion der Schule. Stuttgart 2001,137-167.

Marotzki, W.: Neue kulturelle Vergewisserung: Bildungstheoretische Perspektive des Internet. In: Sandbothe, M./Marotzki, W. (Hrsg.): Subjektivität und Öffentlichkeit. Köln 2000,236-258.

Schäfer, K.-H.: Franz Fischers Bildungskategoriale Pädagogik und die Interaktionspädagogik. In: Franz-Fischer-Kreis: Norderstedter Hefte für Philosophie und Pädagogik, 3. Jg. (1988), Heft 1/2, S. 3-26.

Schreier, H. (Hrsg.): Rekonstruktion der Schule. Stuttgart 2001.

Seufert, S./Back, A./Häusler, M.: E-Learning. Weiterbildung via Internet. Das ,Plato-Cookbook' für internetbasiertes Lernen. Kilchberg 2001.

Simons, P.R.J. (1992): Lernen, selbständig zu lernen - ein Rahmenmodell. In: Mandl/ Friedrich (Hrsg.): Lern- und Denkstrategien - Analyse und Intervention. Göttingen, 251-264. 
Spanhel, D.: Erziehung in einer mediengeprägten Alltagswelt. In: Liedtke, M. (Hrsg.): Kind und Medien. Bad Heilbrunn 1997, S. 229-248.

Wilbers, K.: E-Leaming didaktisch gestalten. In: Hohenstein, A./Wilbers, K. (Hrsg.): Handbuch ELearning. Köln 2001, Kap. 40.

de Witt, C.: Neue Medien und die Pädagogik des Pragmatismus. Dortmund 1999. 